\title{
Methodological approach in the conservation of coastal fortifications
}

\author{
Gianfranco Carcangiu ${ }^{a}$, Stefano Columbu ${ }^{b}$, Paola Melonic, Maura Carboni ${ }^{\mathrm{d}}$, Marta \\ Casti $^{\mathrm{e}}$, Ombretta Cocco ${ }^{\mathrm{e}}$, Arianna Murru ${ }^{\mathrm{a}, \mathrm{e}}$, Marcella Palomba ${ }^{\mathrm{a}}$, Alessandro Ruggieri ${ }^{\mathrm{c}}$ \\ a Consiglio Nazionale delle Ricerche - Istituto di Scienze dell'Atmosfera e del Clima, Bologna, Italy, \\ g.carcangiu@isac.cnr.it, m.palomba@isac.cnr.it, 'bniversità degli Studi di Cagliari, Dipartimento di Scienze \\ Chimiche e Geologiche, Cagliari, Italy, columbus@unica.it ${ }^{c}$ Università degli Studi di Cagliari, Dipartimento di \\ Ingegneria Meccanica, Chimica e dei Materiali, Cagliari, Italy, paola.meloni@unica.it, ruggieri.alessandro@libero.it, \\ ${ }^{\mathrm{d}}$ Università degli Studi di Sassari, Dipartimento di Scienze Chimiche e Farmaceutiche, Sassari, Italy, \\ maucarboni@uniss.it, ${ }^{\circ}$ Dipartimento di Ingegneria Civile, Ambientale e Architettura, University of Cagliari, Italy, \\ marta.casti@unica.it, ombretta.cocco@unica.it, arianna.murru@unica.it
}

\begin{abstract}
The historical and artistic value of Coastal Towers justifies the recovery of these emblematic artifacts. For the achievement of this objective we propose a methodological approach including: i) architectural relief also by 3D laser scanner and collection of historical data; ii) macroscopic semi-quantitative evaluation of the decay, iii) application of diagnostic quantitative chemical-physical-mechanical methodologies, to assess the decay intensity, and define the criticalities for planning the restoration.

The i step allows the graphic rendering of the building showing the geometry and the relationships between the parties. The ii step allows a preliminary understanding of the building materials, macroscopic alteration forms, monument features, acquisition of information about potential causes and decay mechanisms. During the iii step, sampling of building materials is planned. Laboratory analyses, to determine the soluble salts, crystalline phases, porosity as well as micro-textural features of the materials, are carried out by the application of suitable techniques.
\end{abstract}

Keywords: Coastal Tower, Decay, Geomaterials, Chemical-physical characterization, Restoration.

\section{Introduction}

Coastal towers are an interesting and fascinating fragment of the historical heritage of several Mediterranean regions, as well as Sardinia. Their past functional aspects, involving the territory of a region, protection of trade, transmission of military information and surveillance of water sources, fully justifies the need to recover these symbolic artifacts, frequently seriously damaged because of the total lack of preservation actions.

The restoring of these artifacts could expand the historical context related to the past coastal defense system, provide useful information about the ancient building processes and the "microeconomics" inherent their construction. In addition, recoveries can also be considered as a profitable investment if the Towers were included in tourist routes.

To succeed in a task of restoration, which is technically and philologically correct, we should know about construction techniques and architectural features of these buildings. It is need to determine, as fully as possible, the behavior of materials to environmental aggression and define the mechanisms that govern them chemical and physical deterioration along with the assessment of their compatibility 
to protective or consolidating products. This will make it possible to draft a hierarchy of damage categories, so you can tailor interventions based on priorities while minimizing costs. We must also identify the most suitable raw materials for replacement or integration, with particular attention to local recycling materials forming originally the structures and detached by them or raw materials whose presence has been mapped in geological maps or ascertained through appropriate prospecting.

Finally, monitoring the state of conservation of the building will allow to obtain data that can be interpolated in a correct durability function (DF) and compare this one with that obtainable from accelerated degradation tests in laboratory.

\section{Methodological approach}

For recovering hystorical artifacts as Coastal Tower we propose an approach that includes the following steps: $i$ ) architectural relief also by $3 \mathrm{D}$ laser scanner; collection of historical data; ii) macroscopic semi-quantitative evaluation of decay forms, iii) application of diagnostic quantitative chemical-physical-mechanical methodologies, for assessing the decay intensity, and define the criticalities for planning the restoration. Step i) allows the graphic rendering of the building showing the geometry and the relationships between the parties; the analysis of these geometric data allowing immediate assessment and measurement of misalignment, failures, loss or collapse of parts or other phenomena that can be prelude to a static breakdown. Step ii) provides fundamental information about building materials, macroscopic alteration forms, artifact features, origin and mechanisms of decay. All acquired information will be significant and fundamental in the planning of a targeted sampling of building materials, for individuating the most appropriate diagnostic techniques to be used in the characterization of materials and/or monitoring, as well as for defining the most urgent actions for recovery. In this step, the architectural relief of monuments makes up the base for representing distribution of the constituent materials (rock-types, mortars and other materials) and of the forms of decay. During the iii) step, according to the information obtained by the macroscopic mapping of decay, sampling of building materials (natural and artificial stones, mortars, metals, wood) will be planned. It will be fundamental to geo-reference the sampling points and acquire a complete photographic documentation.

Laboratory analyses and in situ tests allow determining: the mineralogical composition, the micro-textural features of the building materials, content of soluble salts and porosity. Other petro-physical properties can be determined by the application of suitable techniques. The chemical-physical-mechanical properties of the collected materials can be used to refine a mapping of materials and decay forms that can be graphically represented on thematic atlantes for each single Tower.

The step ii), following the architectural relief, allows you to recognize and classify the macroscopic decay forms that affect the Tower, so assessing the conservation condition and identifying the criticalities that require urgent interventions. The classification of weathering forms proposed by the Working group "Natural stones and weathering" of the Geological Institut RWTH, Aachen University [1], represents a suitable method to quantify the damage that affect a monument. The effectiveness of the method implies an appropriate scaling of the in situ measurements. This classification is more effective than the UNI EN 1182 [2], because it establishes an ordered hierarchical approach.

The macroscopic investigations have to be carried out by a semi-quantitative methodological approach aimed to recognize: 1) the single decay forms of each building material, 2) the relative extension and intensity (areal, linear and volumetric), and 3) the damage degree, computable according to the so-called "Damage Categories", using statistical estimators, correlation matrices and in situ measurements. From the accuracy of the architectural relief of monument will depend the objective quantification of damage.

In the mapping phase, the creation of a thematic atlas concerning the decay forms for each object 
of interest, can provide an useful support for the prosecution of the investigations.

The synthetic document, resulting by the application of these procedures, will be edited in a false-color map, representing the different Categories of Damage along with linear and progressive damage index that affect the monument. The proposed methodological approach gives several useful advantages: a better understanding of the decay forms for the different types of building materials; features of the monument; information about potential causes and mechanisms of decay; information for planning a targeted sampling of materials to be analyzed; appropriate choice of diagnostic and/or monitoring techniques; determination of the most urgent actions for restoring. Based on information acquired by the mapping, the building materials will be sampled. In this phase the location of the sampling points have to be geo-referenced and an accurate photographic documentation must be acquired.

After this step, analytical methods and tests have to be applied to determine the intrinsic features of materials, as well as state of preservation and presence and type of alteration. The acquired results will allow setting the more appropriate conservation techniques according to the different types of materials and decay.

\section{In situ tests and Laboratory investigations}

Laboratory analyses and tests will be performed in relation to the specific issues.

In the specific case of the Coastal Towers, we suggest the following analyses that revealed the most useful in the investigation of the building materials:

- In situ ND (non-destructive) measurements: ultrasonic, sonic, sclerometric, microdrilling resistance measurements, and thermal infrared investigations can be carried out to assess the physical and mechanical performances.

- Analysis by X-Ray Powder Diffraction (XRPD), for identifying the crystalline phases.

- XRF analysis to determine the chemical composition.
- Thermogravimentric (TG-DTA) and thermodifferential analysis, for identifying the nature of some amorphous or poorly crystalline phases.

- Determination of soluble salts by Ion Chromatography (IC).

- SEM and optical microscopy studies, for defining microstructure, qualitative composition of the mineral phases forming materials, and mixing ratios of mortars.

- Porosimetric investigation, aimed at identifying the porosity of the materials, pore size distribution, skeletal and apparent density.

- Micro FTIR analyses to recognize the presence of restoring products, particulary acrylic, siliconic and fluorurate, applied on the surfaces. Their presence can be interfered on the sorption and effectiveness of a new treatment that can be done.

The final diagnosis, deducted by the critical analysis of whole dataset, is the base for planning the recovery of the building.

In particular, the susceptibility of the materials to stresses induced by salt crystallization [3] and wind erosion [4] will need to be carefully assessed.

Therefore quarry materials similar to those of the towers may be retrieved, in order to perform specific tests.

In particular, the resistance to salt crystallization ( $\mathrm{NaCl}$ and $\mathrm{Na}_{2} \mathrm{SO}_{4} \cdot 10 \mathrm{H}_{2} \mathrm{O}$ aqueous solution) will be evaluated in a climatic chamber, even on samples subject of treatment with consolidating and protective products.

It will be necessary to perform the physicalmechanical characterization of these samples and in particular to verify at least the mechanical strength (compressive and tensile), porosity, abrasion resistance, pulse speed before and after the accelerated degradation in the climate room.

In this regard it is noted that there is no European legislation covering the methods of the treatments and the assessment of its effectiveness. 
Even on tests of accelerated degradation, some aspects are still open [6-7]. There is no legislation governing the conduct of the tests of degradation, even for lime-based mortars or NHL frequently used in the conservation field.

The outcome of the experiments (ex ante and post treatment) and the information derived from the application of the protocol, should allow specifying some specific aspects of the restoration project (the nature of the repair mortar, consolidating and protective products for stone).

At the same time the processing of the data can be converted into a durability factor (DF) for different materials, or also for the structure and predict the useful life time.

Therefore actions targeted to monitoring and specific maintenance programs can be developed. The latter are regularly disregarded while they would reduce the costs, compared to those that are reached in an emergency situation (risk of collapse), allow small companies to perform sustainable restoration work, philologically consistent and respectful of the matter.

\section{Conclusions}

Data and interpretations of the results of investigations aimed at the characterization of building materials will constitute a collection, organized as cards for each Tower to be restored, for easy transposition in relational databases.

These cards can be a useful reference for similar actions, and summarize the results obtained by the application of analytical techniques and tests. The analysis of decay, the study of diseases of artifacts, the complete acquisition of chemicalphysical features of building materials, are essential to make choices technically and philologically correct.

\section{References}

Fitzner B., Heinrichs K., La Bouchardiere D. (2003), Weathering damage on Pharaonic sandstone monuments in Luxor-Egypt. Building and Environment, 38, 9-10, pp. 1089-1103.

UNI EN 1182:2006 .Cultural Heritage; Natural and artificial stone. Description of the alterations Terminology and definition.

Rodriguez-Navarro C., Doehne E. (1999), Salt weathering: influence of evaporation rate, supersaturation and crystallization pattern. Earth Surface Processes and Landforms 24, pp. 191209.

Camuffo D. (1993), Controlling the aeolian erosionof the great Sphinx. Studies in Conservation 38, pp. 198-205.

Camuffo D. (1998), Microclimate for cultural Heritage. Ed. Elsevier, 225 Wyman Street, Waltham, MA 02451 USA.

Moropoulou A., Kouloumbi N., Haralampopoulos G., Konstanti A., Michailidis P. (2003), Criteria and methodology for the evaluation of conservation interventions on treated porous stone susceptible to salt decay. Progress in Organic Coating, 48, 2-4, pp. 259-270.

Matthieu Angeli, Benavente D., Bigas J.P., Menéndez B., Hébert R., David C. (2008), Modification of the porous network by salt crystallization in experimentally weathered sedimentary stones. Materials and Structures, 41, 6, pp. 1091-1108. 